\title{
Factors associated with motor vehicle-related eye injuries presenting to the US emergency departments
}

\author{
Sunali Goyall ${ }^{1,2,3} \cdot$ Mallikarjuna Rettiganti $^{4}$ Punkaj Gupta ${ }^{4,5}$ \\ Received: 20 April 2018 / Accepted: 1 September 2019 / Published online: 25 September 2019 \\ (c) The Author(s), under exclusive licence to The Royal College of Ophthalmologists 2019
}

\begin{abstract}
Objectives To evaluate risk factors associated with eye injuries related to motor vehicle accidents (MVA) using a multicentre national database from the US emergency departments.

Patients and methods A post hoc analysis from the Nationwide Emergency Department Sample database (2006-2013) was performed. Patients presenting to the emergency department (ED) with MVA-related trauma were included. We then evaluated patient- and centre-level risk factors associated with the presence of ocular trauma using a multivariable logistic regression model.

Results A total of 2,745,152 patients from 2080 ED were identified for inclusion. Of these, 31,493 patients (1.14\%) were associated with ocular trauma. The most common ocular injuries reported in our study were injury to ocular adnexa, orbital fractures, subconjunctival haemorrhage, foreign body on eye, and open wound of the eyeball. In univariate analysis, the other patient factors associated with ocular trauma included male gender, $>1$ chronic condition, admission during weekend, $>1$ bodily injury, higher injury severity score (ISS), presence of skull fractures and other bodily fractures, seizures, and initiation of mechanical ventilation after MVA. The hospital characteristics associated with highest incidence of ocular trauma included ED visit to a level I trauma centre and metropolitan teaching hospital. The independent factors associated with ocular trauma included younger age groups, male gender, weekend injury, presence of chronic conditions, $>1$ bodily injury, higher ISS, presence of skull fractures, ED admission to level-1 trauma centre, and ED admission to a teaching hospital.
\end{abstract}

Conclusions This multicentre study establishes that both patient- and centre-level factors are associated with MVA-related eye injuries.

Supplementary information The online version of this article (https:// doi.org/10.1038/s41433-019-0588-7) contains supplementary material, which is available to authorized users.

Sunali Goyal

sunali_goyal@hotmail.com

1 Department of Ophthalmology, The University of Texas Health Science Center, San Antonio, TX, USA

2 Department of Ophthalmology, University of Arkansas for Medical Sciences, Little Rock, AR, USA

3 Harvey and Bernice Jones Eye Institute, Little Rock, AR, USA

4 Department of Pediatrics, University of Arkansas for Medical Sciences, Little Rock, AR, USA

5 Section of Cardiac Critical Care, Methodist Children's Hospital, San Antonio, TX, USA

\section{Introduction}

Motor vehicle accidents (MVAs) remain a leading cause of morbidity and mortality in the United States (US). MVAs predispose drivers and passengers to an enhanced risk for ocular injuries. The incidence of MVA-related eye injuries has been reported to vary from 0.2 to $3.4 \%$ [1-5]. An estimated 9280-11,600 eyeball injuries occur in the USA as a result of MVAs annually [2]. The ocular injuries in MVAs can range from superficial injuries, such as minor eyelid injury or corneal abrasion, to potentially blinding injuries, such as globe rupture or lens dislocation [1-5]. Some of the factors that lead to ocular injuries in MVAs include sudden deceleration, air bag deployment, broken glass, ejection from the vehicle, etc [1-5]. Crashes of increased severity with larger changes in velocity have been associated with an increased risk of MVA-related eye injuries $[3,4]$. 
It has been suggested that majority of MVA-related eye injuries occurring in the USA are seen in ED [1]. However, there are no studies evaluating the risk factors associated with MVA-related eye injuries among patients presenting to the US emergency departments. To address this knowledge gap, we designed this study to evaluate patient- and centrelevel risk factors associated with MVA-related eye injuries using a multicentre national database from the US emergency departments, the Nationwide Emergency Department Sample (NEDS).

\section{Methods}

\section{Data source}

NEDS is a set of hospital-based emergency department (ED) databases included in the Healthcare Cost and Utilization Project (HCUP) family [6]. It is the largest allpayer ED database in the USA and includes data from billing records of 25-30 million ED visits across all age groups from more than 950 hospitals across the USA. NEDS is a part of a federal, state, and private industry partnership project that has been developed to aid decision-making at the national, state, and community levels [7]. NEDS data is sampled from State Inpatient Databases that include information on the patients seen in the ED and then admitted to the same hospital and from State Emergency Department Databases, which capture ED visits not requiring admission. There are about 130 million visits to the ED each year in the USA, and each case is assigned a weight. Poststratification weights then allow estimation of the national data. NEDS includes a number of variables for each hospital stay such as the International Classification of Diseases, Ninth Revision, Clinical Modification (ICD-9) codes; Current Procedure Terminology, Fourth Edition (CPT) procedure codes; admission and discharge status; patient demographic; and expected payment charges. NEDS has extensive quality control procedures. The datafile related to quality control for NEDS can be accessed from the following link: https://www.hcup-us.ahrq.gov/db/quality.pdf. NEDS is part of the family of databases and software tools developed for the HCUP. HCUP was created to provide a robust source of health care data that could be used to further research, improve health care, and inform decision making. Administrative data sets provide a readily available source of "real-world" health care data on a large population of unselected patients. Because of the sheer numbers of patients included in databases such as the NEDS, the data are considered to be representative of the populations of interest.

\section{Study population}

This analysis focused on the de-identified data for ED visits in NEDS for the period January 2006 to December 2013. We identified individuals presenting to the ED with MVArelated trauma utilizing ICD-9 and CPT codes. The study population was divided in two study groups: (1) ocular trauma group, in which the patients were associated with MVA-related eye injuries, and (2) no ocular trauma group, in which the patients were not associated with any MVArelated eye injuries. No specific exclusion criteria were applied to the study population. The current study was granted exempt status from the Institutional Review Board at the University of Arkansas for Medical Sciences and adhered to the Declaration of Helsinki and all federal and state laws.

\section{Data collection}

Several variables of interest were studied. Table 1 provides a detailed list of ICD-9 codes related to eye injuries included in this study. Data collected included demographic information (such as age and gender), underlying chronic conditions, and month of ED visit. We also collected data on diagnoses and injuries related to MVAs such as seizures (ICD-9, 345.xx, 780.31-39, 779.0), skull fractures (ICD-9, 802.xx, 803.xx, 804.xx), other body fractures (ICD-9, 805.xx, 806.xx, 807.xx, 808.xx, 809.xx), and interventions such as mechanical ventilation (ICD-9, 96.70, 96.71, 96.72, or CPT, 94002, 94003, 94656). Patients were also identified and grouped according to injury severity score (ISS) [8]. ISS is an established medical score to assess and define trauma severity. It is an anatomical scoring system that is used to calculate an overall score for patients with multiple injuries and is the only score that correlates linearly with mortality, morbidity, hospital stay, and other measures of severity. The overall ISS ranges from 0 to 75. A major trauma is defined as an ISS of $>15$. We specified several other facility and visit variables including the primary payer involved, teaching status of the hospital, and the complexity level of the trauma centre. We also collected data on disposition after ED treatment, such as whether patients were treated and released, transferred to another facility, or admitted to the same hospital, and final disposition including death. The intracluster correlation coefficient for study variables are depicted in e-Table 1 of the online supplement. The levels of trauma centre are explained in the online supplement.

\section{Statistical analysis}

The purpose of the statistical analysis was to identify factors associated with the presence of ocular trauma. 
Table 1 Diagnoses related to ocular trauma used in our study population

\begin{tabular}{llll}
\hline Type of ocular trauma & ICD-9 codes & $N=31,493$ & Frequency in MVA \\
\hline Open wound of ocular adnexa & $870 . x x$ & 8993 & 33 in 10,000 \\
Contusion of eye and adnexa & $921 . x x$ & 8111 & 30 in 10,000 \\
Superficial injury of eye and adnexa & $918 . x x$ & 6618 & 24 in 10,000 \\
Orbital fractures & $802.6 \times$ & 6306 & 23 in 10,000 \\
Subconjunctival hemorrhage & 372.72 & 2565 & 9 in 10,000 \\
Foreign body on external eye & $930 . x x$ & 1269 & 5 in 10,000 \\
Open wound of eyeball & $871 . x x$ & 707 & 3 in 10,000 \\
Orbital hemorrhage & 376.32 & 599 & 2 in 10,000 \\
Optic nerve injury & $950 . x x$ & 202 & 7 in 1000 \\
Vitreous hemorrhage & 379.23 & 109 & 4 in 1000 \\
Burns confined to eye and adnexa & $940 . x x$ & 108 & 4 in 1000 \\
Other ocular nerve injuries & $951.0,951.1,951.3$ & 102 & 4 in 1000 \\
Retinal detachment & $361 . x x$ & 81 & 3 in 1000 \\
Hyphema of iris and ciliary body & 364.41 & 63 & 2 in 1000 \\
Retinal hemorrhage & 362.81 & 61 & 2 in 1000 \\
Choroidal hemorrhage (or rupture) & $363.61,363.62,363.63$ & 22 & 1 in 1000 \\
Iridodialysis & 364.76 & 22 & 1 in 1000 \\
Traumatic cataract & $366.2 \times$ & 22 & 1 in 1000 \\
Endophthalmitis & $360.00,360.01$ & 15 & 1 in 1000 \\
Optic nerve hemorrhage & 377.42 & 2 & 0 \\
\hline
\end{tabular}

ICD International Classification of Diseases, $M V A$ motor vehicle accident
We summarized patient-and centre-level factors using appropriate summary statistics such as mean and standard deviation or frequency and percentages. A multivariable logistic regression model was used to identify factors associated with ocular trauma. We performed chi square test for association to check for collinearity between variables and variables that were significantly correlated with each other were simultaneously not included in the model.

Model selection was performed using a logistic adaptive LASSO model with AICc validation. The LASSO model penalizes variables for multicollinearity and the variables that did not drop out from the LASSO model were included in the final logistic regression model. The coefficients for head trauma (yes/no) and other fractures (yes/no) were shrunk to zero in the LASSO model and thus were not included in the final model. The following variables finally included in the multivariable logistic regression model were: age, gender, weekend admission (yes/no), groups based on ISS $(0,1,2-3$, and $\geq 4$ ), more than 1 chronic condition (yes/no), multiple injuries (yes/no), seizures (yes/no), skull fractures (yes/no), other fractures (yes/no), mechanical ventilation (yes/no), discharge quarter (January-March, April-June, July-September, October-December), primary payer (Medicare, Medicaid, private including HMO, Self-Pay/Other), hospital trauma level (nontrauma, level I, level II, level III), and hospital teaching status (metropolitan nonteaching, metropolitan teaching, and nonmetropolitan). Interactions between age groups and gender, $>1$ bodily injury and injury severity group, $>1$ bodily injury and skull fractures, and $>1$ bodily injury and mechanical ventilation were considered for inclusion in the model. However, they were either not statistically significant or clinically meaningful. Therefore, the final model did not include any interactions among variables in the logistic regression models. Missing data were very minimal across all variables $(<1 \%)$ and therefore a complete case analysis was done and the analysis was not expected to have any significant bias due to omission of observations with missing data. The effects of predictors on ocular trauma are shown using odds ratios and $95 \%$ confidence intervals. Further, we show standardized beta coefficients from the logistic regression model as a means of showing effects sizes. Standardized coefficients enable comparison of the effect of one variable compared with the other and eliminates the influence of sample size on the significance of the variable. The area under the ROC curve for the final model was 0.80 suggesting the model has high predictive ability in distinguishing ocular trauma patients from those who do not.

The adaptive logistic LASSO model for variable selection was performed using JMP software version 14 (SAS Institute Inc). All other analyses were done using the SAS/STAT software, Version 9.4 of the SAS System for Windows. All plots were generated using the ggplot2 package in $\mathrm{R}$ ( $\mathrm{R}$ Core Team, Vienna, Austria). In each model, discharge weights were used for each patient to 
make the results generalizable to all patients. $P$-values $\leq$ 0.05 were considered to be statistically significant.

\section{Results}

A total of 2,745,152 patients from 2080 ED were identified with MVAs during the study period. From this group, 31,493 patients $(1.14 \%)$ were associated with ocular trauma. Overall, MVAs were more common in females $(53.1 \%)$ and more common in the age groups $19-35$ years $(40.1 \%)$ and $36-60$ years $(33.1 \%)$. The most common ISS was $1(41.6 \%)$, and the mean (standard deviation) ISS for the study patients was 2.5 (3.9). The majority of MVArelated patients had only one or no injury diagnoses reported (55.8\%). The highest incidence of MVA was noted in metropolitan teaching hospitals $(45.6 \%)$, followed by metropolitan nonteaching hospitals (42.8\%). Surprisingly, nontrauma centres were associated with the highest incidence of MVA (57.9\%). The majority of all MVA-related patients were treated and released from ED (91.5\%).

The most common ocular injuries reported in our study were injury to ocular adnexa, orbital fractures, subconjunctival haemorrhage, foreign body on eye, and open wound of the eyeball (Table 1). Ocular trauma was more commonly noted in the age groups 19-35 years, followed by the age group 36-60 years (Table 2). Although MVAs were more commonly noted among females $(53 \%)$, ocular trauma was more commonly noted in males (62\%). In univariate analysis, the other patient factors associated with ocular trauma included $>1$ chronic condition, admission during weekend, $>1$ bodily injury, higher ISS, presence of skull fractures and other bodily fractures, seizures, and initiation of mechanical ventilation after an MVA. Oculartrauma patients were uniformly distributed across different months of the year without any seasonal association. The hospital characteristics associated with highest incidence of ocular trauma included ED visits to a Level I trauma centres and metropolitan teaching hospitals (Fig. 1). Approximately one-third of the MVA patients were admitted to the same hospital after ocular trauma.

We performed multivariable logistic regression model to select independent factors associated with ocular trauma. Due to the large sample size in our study, it was possible that factors that are not clinically relevant could turn out to be statistically significant [9]. To offset the effect of sample size, we used a cutoff of standardized coefficient $\geq 0.05$ to determine independent factors associated with ocular trauma after an MVA. The independent factors associated with ocular trauma included younger age group (9-18 years vs. $<1$ year, odds ratio, OR: $0.78,95 \%$ confidence interval, CI: 0.73-0.84), male gender (female vs. male, OR: 0.73, 95\% CI: 0.72-0.74), weekend injury (yes vs. no, OR: 1.17 ,
95\% CI: 1.16-1.18), presence of chronic conditions (>1 vs. $\leq 1$, OR: $1.31,95 \%$ CI: $1.30-1.34$ ), $>1$ bodily injury (yes vs. no, OR: 4.63 , 95\% CI: $4.55-4.72)$, higher ISS (4-75 vs. 0 , OR: 4.12 , 95\% CI: 3.93-4.32), presence of skull fractures (yes vs. no, OR: 9.49, 95\% CI: 9.35-9.64), ED admission to level-1 trauma centre (level 1 trauma centre vs. nontrauma centre, OR: 2.05, 95\% CI: 2.01-2.08), and ED admission to a teaching hospital (nonmetropolitan vs. metropolitan nonteaching, OR: 1.24, OR: 1.23-1.27) (Table 3). In simple terms, presence of skull fracture is associated with $849 \%$ increase risk of ocular trauma, or weekend injury is associated with $17 \%$ increased risk of ocular trauma. The variables removed from this model were month of ED visit, seizures, other bodily fractures, initiation of mechanical ventilation after an MVA, and primary payer status. The $p$-value for the Hosmer Lemeshow goodness of fit test for our model was 0.34 , indicating good calibration.

\section{Discussion}

Our study reports patient- and centre-level factors associated with MVA-related eye injuries using a large, diverse patient population from 2080 US emergency departments. The independent factors associated with ocular trauma included younger age group, male gender, weekend injury, presence of chronic conditions, $>1$ bodily injury, higher ISS, presence of skull fractures, ED admission to level-1 trauma centre, and ED admission to a teaching hospital. Our study reports an incidence rate of 11.47 per 1000 population for MVA-related eye injuries. This rate is similar to reported incidence in existing literature [1-5]. In comparison to other studies, our study utilized a more recent study period and more extensive eye-trauma-related diagnoses categories to report rate of MVA-related eye injuries.

Our study demonstrated higher probability of ocular trauma among males as compared with females. It is possible that increased risk-taking behaviour and aggressive driving in males could have contributed to this increased probability of ocular trauma [10]. We also noted lower probability of ocular trauma among older children and adults as compared with younger children. We also noted that patients with $>1$ bodily injury and patients with higher ISS were associated with higher probability of ocular trauma after MVA. To our knowledge, no previous study has made this direct correlation between ISS score and likelihood of ocular trauma.

Another factor strongly correlated with ocular trauma included presence of skull fractures after MVA. This association is in accordance with many previous studies that have shown that ocular trauma was more likely to occur in patients with midfacial fractures, especially if there were multiple fractures $[11,12]$. Other studies have examined the 
Table 2 Patient and center characteristics

\begin{tabular}{|c|c|c|c|c|}
\hline & $N$ & $\begin{array}{l}\text { No ocular trauma } \\
N=2,713,659(\%)\end{array}$ & $\begin{array}{l}\text { Ocular trauma } \\
N=31,493(\%)\end{array}$ & $P$ \\
\hline Age (years) & $2,745,141$ & & & $<0.001$ \\
\hline$<1$ & & $27,573(1.0 \%)$ & $191(0.6 \%)$ & \\
\hline $1-8$ & & $100,800(3.7 \%)$ & $1321(4.2 \%)$ & \\
\hline $9-18$ & & $328,678(12.1 \%)$ & $4536(14.4 \%)$ & \\
\hline $19-35$ & & $1,088,461(40.1 \%)$ & $12,840(40.8 \%)$ & \\
\hline $36-60$ & & $900,286(33.2 \%)$ & $9418(29.9 \%)$ & \\
\hline $61-80$ & & $227,951(8.4 \%)$ & $2612(8.3 \%)$ & \\
\hline$>80$ & & $39,899(1.5 \%)$ & $575(1.8 \%)$ & \\
\hline Female gender & $2,744,071$ & $1,443,392(53.2 \%)$ & $11,997(38.1 \%)$ & $<0.001$ \\
\hline$>1$ chronic condition & $2,744,951$ & $385,174(14.2 \%)$ & $7880(25.0 \%)$ & $<0.001$ \\
\hline Months of ED visit & $2,744,313$ & & & 0.19 \\
\hline January-March & & $619,079(22.8 \%)$ & $7105(22.6 \%)$ & \\
\hline April-June & & $695,457(25.6 \%)$ & $8012(25.4 \%)$ & \\
\hline July-September & & $702,569(25.9 \%)$ & $8126(25.8 \%)$ & \\
\hline October-December & & $695,726(25.6 \%)$ & $8239(26.2 \%)$ & \\
\hline Admission during weekend & $2,744,594$ & $729,269(26.9 \%)$ & $10,693(34.0 \%)$ & $<0.001$ \\
\hline$>1$ bodily injury & $2,745,152$ & $1,187,915(43.8 \%)$ & $26,587(84.4 \%)$ & $<0.001$ \\
\hline Injury severity group & & & & $<0.001$ \\
\hline 0 & & $377,752(13.9 \%)$ & $472(1.5 \%)$ & \\
\hline 1 & & $1,135,732(41.9 \%)$ & $6251(19.9 \%)$ & \\
\hline $2-3$ & & $603,645(22.3 \%)$ & $7162(22.8 \%)$ & \\
\hline $4-75$ & & $593,784(21.9 \%)$ & $17,578(55.9 \%)$ & \\
\hline Seizures & $2,745,152$ & $20,932(0.8 \%)$ & $462(1.5 \%)$ & $<0.001$ \\
\hline Skull fractures & $2,745,152$ & $29,190(1.1 \%)$ & $7124(22.6 \%)$ & $<0.001$ \\
\hline Other fractures & $2,745,152$ & $133,839(4.9 \%)$ & $5716(18.2 \%)$ & $<0.001$ \\
\hline Mechanical ventilation & $2,745,152$ & $13,566(0.5 \%)$ & $1236(3.9 \%)$ & $<0.001$ \\
\hline Trauma level & $2,745,152$ & & & $<0.001$ \\
\hline Nontrauma center & & $1,578,903(58.2 \%)$ & $11,537(36.6 \%)$ & \\
\hline Level 1 center & & $527,762(19.4 \%)$ & $12,135(38.5 \%)$ & \\
\hline Level 2 center & & $384,834(14.2 \%)$ & $5725(18.2 \%)$ & \\
\hline Level 3 center & & $222,160(8.2 \%)$ & $2096(6.7 \%)$ & \\
\hline Hospital teaching status & $2,745,152$ & & & $<0.001$ \\
\hline Metropolitan nonteaching & & $1,165,708(43.0 \%)$ & $9156(29.1 \%)$ & \\
\hline Metropolitan teaching & & $1,233,157(45.4 \%)$ & $19,157(60.8 \%)$ & \\
\hline Nonmetropolitan & & $314,794(11.6 \%)$ & $3180(10.1 \%)$ & \\
\hline Primary payer & $2,730,407$ & & & 0.01 \\
\hline Medicare & & $109,787(4.1 \%)$ & $1357(4.3 \%)$ & \\
\hline Medicaid & & $321,358(11.9 \%)$ & $3790(12.1 \%)$ & \\
\hline Private including HMO & & $1,437,574(53.3 \%)$ & $16,678(53.4 \%)$ & \\
\hline Self-pay/other & & $830,443(30.8 \%)$ & $9420(30.1 \%)$ & \\
\hline Disposition (after ED treatment) & $2,745,152$ & & & $<0.001$ \\
\hline Patient treated and released & & $2,492,097(91.8 \%)$ & $19,169(60.9 \%)$ & \\
\hline Patient admitted to same hospital & & $173,933(6.4 \%)$ & $10,938(34.7 \%)$ & \\
\hline $\begin{array}{l}\text { Patient transferred to other short- } \\
\text { term hospital }\end{array}$ & & $36,236(1.3 \%)$ & $1210(3.8 \%)$ & \\
\hline Patient died in the ED & & $5688(0.2 \%)$ & $94(0.3 \%)$ & \\
\hline Other & & $5705(0.2 \%)$ & $82(0.3 \%)$ & \\
\hline
\end{tabular}

$E D$ emergency department, $H M O$ health maintenance organization 

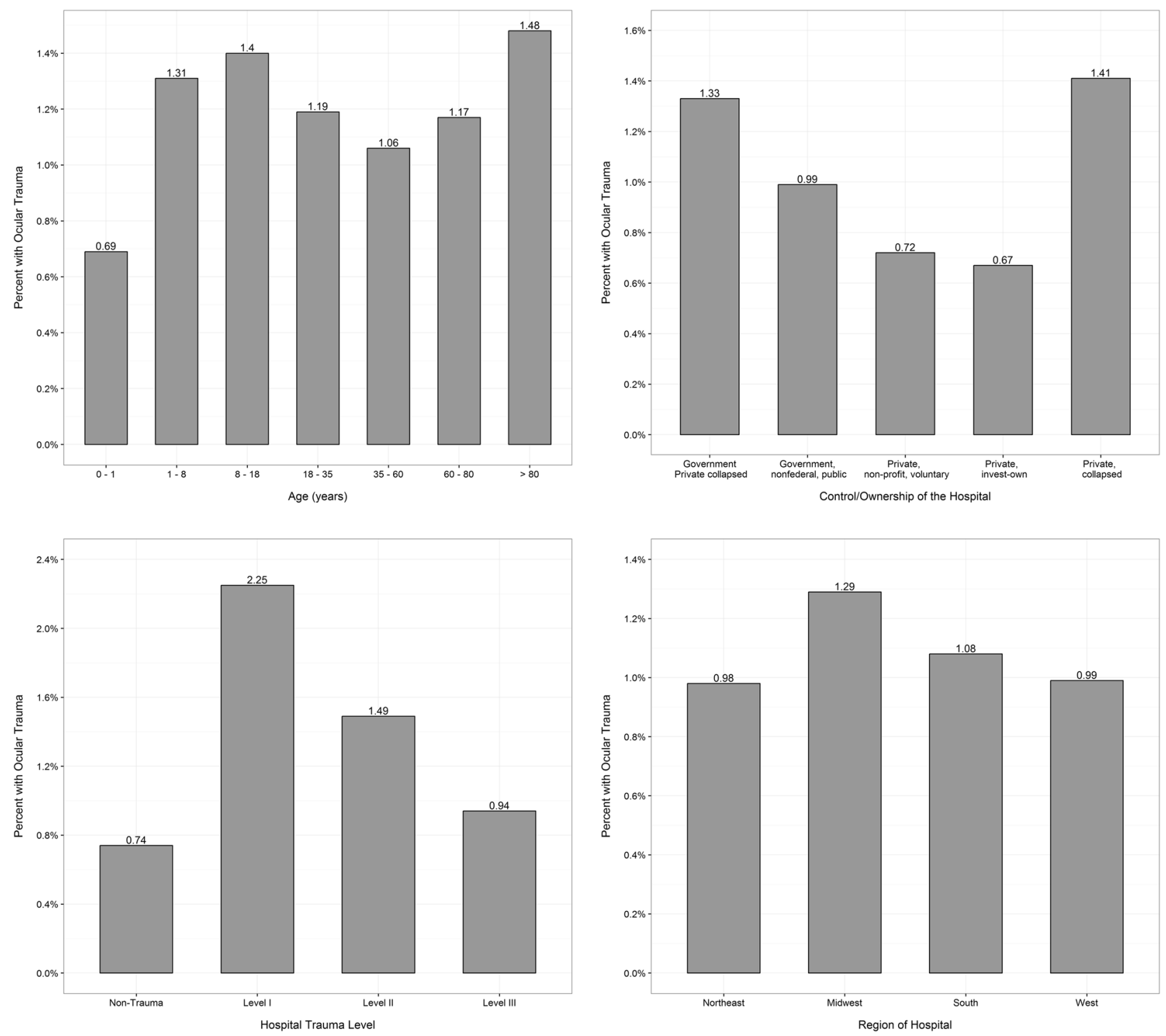

Fig. 1 Distribution of MVA-related ocular trauma by age, control/ownership of the hospital, hospital trauma level, and region

effect of airbags in orbital fractures in MVAs [3, 4]. As expected, we noted higher probability of ocular trauma among patients presenting to level 1 trauma centres. Surprisingly, nonmetropolitan hospitals were associated with higher probability of ocular trauma. As these hospitals were located in suburban or rural areas, it is possible that the patients presenting to these hospitals were associated with increased use of heavy vehicles or were associated with increased risk-taking behaviours that led to higher incidence of ocular trauma.

Our study has several limitations. The retrospective nature of our study renders it susceptible to study design flaws and bias. Although we attempted to adjust for important patient- and centre-level variables, it is possible our dataset lacked important confounders that could have impacted our analysis. Another limitation of this study is the use of an administrative database for case ascertainment. Misclassification bias may have been introduced because many ICD-9-related conditions lack explicit definitions. They are also subject to errors at multiple points, including diagnostic errors, communication errors, and transcription errors. The ICD-9 codes may be limited by the level of detail they provide, thus resulting in a lack of granularity.

It is easy not to record eye injury cases occurring as a secondary diagnosis due to trauma or missed when coded under an umbrella of a general diagnosis, so the data potentially are skewed toward underestimation of the actual eye injuries that may have occurred. Also, these injuries only include patients presenting to the ED. We may be missing patients seeking care to non-ED healthcare 
Table 3 Factors associated with MVA related eye injuries

\begin{tabular}{|c|c|c|c|c|}
\hline Variable & Coefficient (SE) & Standardized coefficient & Odds ratio $(95 \% \mathrm{CI})$ & $P$ \\
\hline \multicolumn{5}{|l|}{ Age (years) } \\
\hline$<1$ & & & Reference & \\
\hline $1-8$ & $0.1130(0.0381)$ & 0.0252 & $1.12(1.04,1.21)$ & 0.003 \\
\hline $9-18$ & $-0.2439(0.0366)$ & -0.0937 & $0.78(0.73,0.84)$ & $<0.001$ \\
\hline $19-35$ & $-0.4849(0.0362)$ & -0.2792 & $0.61(0.57,0.66)$ & $<0.001$ \\
\hline $36-60$ & $-0.6965(0.0364)$ & -0.3850 & $0.49(0.46,0.54)$ & $<0.001$ \\
\hline $61-80$ & $-0.6840(0.0375)$ & -0.2230 & $0.50(0.47,0.54)$ & $<0.001$ \\
\hline$>80$ & $-0.5636(0.0417)$ & -0.0799 & $0.56(0.53,0.62)$ & $<0.001$ \\
\hline \multicolumn{5}{|l|}{ Gender } \\
\hline Male & & & Reference & \\
\hline Female & $-0.3135(0.0057)$ & -0.1839 & $0.73(0.72,0.74)$ & $<0.001$ \\
\hline \multicolumn{5}{|l|}{ Months of ED visit } \\
\hline January-March & & & Reference & \\
\hline April-June & $-0.0393(0.0079)$ & -0.0202 & $0.96(0.94,0.97)$ & $<0.001$ \\
\hline July-September & $-0.0566(0.0079)$ & -0.0291 & $0.94(0.93,0.96)$ & $<0.001$ \\
\hline October-December & $0.0253(0.0078)$ & 0.0130 & $1.02(1.01,1.04)$ & 0.001 \\
\hline \multicolumn{5}{|l|}{ Weekend injury } \\
\hline No & & & Reference & \\
\hline Yes & $0.1560(0.0058)$ & 0.0814 & $1.17(1.16,1.18)$ & $<0.001$ \\
\hline \multicolumn{5}{|l|}{ Chronic conditions } \\
\hline$\leq 1$ & & & Reference & \\
\hline$>1$ & $0.2753(0.0072)$ & 0.1134 & $1.31(1.30,1.34)$ & $<0.001$ \\
\hline \multicolumn{5}{|l|}{$>1$ bodily injury } \\
\hline No & & & Reference & \\
\hline Yes & $1.5339(0.0096)$ & 0.8952 & $4.63(4.55,4.72)$ & $<0.001$ \\
\hline \multicolumn{5}{|l|}{ Injury group } \\
\hline 0 & & & Reference & \\
\hline 1 & $1.1486(0.0228)$ & 0.6651 & $3.15(3.02,3.30)$ & $<0.001$ \\
\hline $2-3$ & $0.8369(0.0243)$ & 0.4082 & $2.30(2.20,2.42)$ & $<0.001$ \\
\hline $4-75$ & $1.4160(0.0238)$ & 0.6947 & $4.12(3.93,4.32)$ & $<0.001$ \\
\hline \multicolumn{5}{|l|}{ Seizures } \\
\hline No & & & Reference & \\
\hline Yes & $0.1320(0.0237)$ & 0.0137 & $1.14(1.08,1.19)$ & $<0.001$ \\
\hline \multicolumn{5}{|l|}{ Skull fractures } \\
\hline No & & & Reference & \\
\hline Yes & $2.251(0.0076)$ & 0.3059 & $9.49(9.35,9.64)$ & $<0.001$ \\
\hline \multicolumn{5}{|l|}{ Other fractures } \\
\hline No & & & Reference & \\
\hline Yes & $0.0703(0.0084)$ & 0.0183 & $1.07(1.05,1.09)$ & $<0.001$ \\
\hline \multicolumn{5}{|l|}{ Mechanical ventilation } \\
\hline No & & & Reference & \\
\hline Yes & $0.1783(0.016)$ & 0.0154 & $1.19(1.15,1.23)$ & $<0.001$ \\
\hline \multicolumn{5}{|l|}{ Trauma level } \\
\hline Nontrauma center & & & Reference & \\
\hline Level 1 center & $0.7156(0.0094)$ & 0.3374 & $2.05(2.01,2.08)$ & $<0.001$ \\
\hline Level 2 center & $0.4449(0.0094)$ & 0.1826 & $1.56(1.53,1.59)$ & $<0.001$ \\
\hline Level 3 center & $0.1538(0.0103)$ & 0.0559 & $1.17(1.14,1.19)$ & $<0.001$ \\
\hline \multicolumn{5}{|l|}{ Hospital teaching status } \\
\hline Metropolitan nonteaching & & & Reference & \\
\hline Metropolitan teaching & $-0.0200(0.0085)$ & -0.0118 & $0.98(0.96,1.00)$ & 0.01 \\
\hline Nonmetropolitan & $0.2217(0.0097)$ & 0.0849 & $1.24(1.23,1.27)$ & $<0.001$ \\
\hline \multicolumn{5}{|l|}{ Primary payer } \\
\hline Private including $\mathrm{HMO}$ & & & Reference & \\
\hline Medicare & $-0.0134(0.0144)$ & -0.0031 & $0.98(0.95,1.01)$ & 0.35 \\
\hline Medicaid & $-0.0251(0.0089)$ & -0.0096 & $0.97(0.95,0.99)$ & 0.004 \\
\hline Self-pay/other & $0.0074(0.0063)$ & 0.0040 & $1.00(0.99,1.02)$ & 0.24 \\
\hline
\end{tabular}

$S E$ standard error, $C I$ confidence interval, $E D$ emergency department, $H M O$ health maintenance organization 
providers. In addition, the study did not classify injuries based on driver and passenger, air bag deployment status, or the setting in which they occurred.

\section{Conclusions}

In this study, we identify risk factors associated with MVArelated eye injuries using a large, diverse patient population from 2080 US emergency departments. The independent factors associated with ocular trauma included younger age groups, male gender, weekend injury, presence of chronic conditions, $>1$ bodily injury, higher ISS, presence of skull fractures, ED admission to level-1 trauma centre, and ED admission to a teaching hospital. Our study provides an opportunity to ED physicians to identify ocular injury and possible timely ophthalmology consultation in patients with MVA injuries.

\section{Salient findings}

(1) Our study reports patient- and centre-level factors associated with MVA-related eye injuries using a large, diverse patient population from 2080 US emergency departments.

(2) Our study reports an incidence rate of 11.47 per 1000 population for MVA-related eye injuries.

(3) In comparison to other studies, our study utilized a more recent study period and more extensive eyetrauma-related diagnoses categories to report rate of MVA-related eye injuries.

(4) The independent factors associated with ocular trauma included younger age group, male gender, weekend injury, presence of chronic conditions, $>1$ bodily injury, higher ISS, presence of skull fractures, ED admission to level-1 trauma centre, and ED admission to a teaching hospital.

(5) Our study provides an opportunity to ED physicians to identify ocular injury and possible timely ophthalmology consultation in patients with MVA injuries.

\section{Summary}

\section{What was known before}

- The incidence of MVA-related eye injuries has been reported to vary from 0.2 to $3.4 \%$. An estimated 9280-11,600 eyeball injuries occur in the USA as a result of MVAs annually.

\section{What this study adds}

- Our study reports patient- and centre-level factors associated with MVA-related eye injuries using a large, diverse patient population from 2080 US emergency departments.

- The independent factors associated with ocular trauma included younger age group, male gender, weekend injury, presence of chronic conditions, $>1$ bodily injury, higher ISS, presence of skull fractures, ED admission to level-1 trauma centre, and ED admission to a teaching hospital.

\section{Compliance with ethical standards}

Conflict of interest The authors declare that they have no conflict of interest.

Publisher's note Springer Nature remains neutral with regard to jurisdictional claims in published maps and institutional affiliations.

\section{References}

1. Armstrong GW, Chen AJ, Linakis JG, Mello MJ, Greenberg PB. Motor vehicle crash-associated eye injuries presenting to U.S. emergency departments. West J Emerg Med. 2014;15:693-700.

2. McGwin G, Owsley C. Risk factors for motor vehicle collisionrelated eye injuries. Arch Ophthalmol. 2005;123:89-95.

3. Duma SM, Rath AL, Jernigan MV, Stitzel JD, Herring IP. The effects of depowered airbags on eye injuries in frontal automobile crashes. Am J Emerg Med. 2005;23:13-19.

4. Duma SM, Jernigan MV, Stitzel JD, Herring IP, Crowley JS, Brozoski FT, et al. The effect of frontal air bags on eye injury patterns in automobile crashes. Arch Ophthalmol. 2002;120:1517-22.

5. Hansen GA, Stitzel JD, Duma SM. Incidence of elderly eye injuries in automobile crashes: the effects of lens stiffness as a function of age. Annu Proc Assoc Adv Automot Med. 2003;47:147-63.

6. HCUP Nationwide Emergency Department Sample (NEDS). Healthcare Cost and Utilization Project (HCUP). Rockville, MD: Agency for Healthcare Research and Quality; (2007-2009). http://www.hcup-us.ahrq.gov/nedsoverview.jsp. Accessed $30 \mathrm{Sep}$ 2017.

7. Introduction to the HCUP Nationawide Emergency Department Sample (NEDS). 2012. http://www.hcup-us.ahrq.gov/db/nation/ neds/NEDS_Introduction_2012.jsp. Accessed 30 Sep 2017.

8. Baker SP, O'Neill B, Haddon W Jr, Long WB. The injury severity score: a method for describing patients with multiple injuries and evaluating emergency care. J Trauma. 1974;14:187-96.

9. Nieminen P, Lehtiniemi H, Vähäkangas K, Huusko A, Rautio A. Standardised regression coefficient as an effect size index in summarising findings in epidemiological studies. Epidemiol Biostat Public Health 2013;2:10.

10. Tavris DR, Kuhn EM, Layde PM. Age and gender patterns in motor vehicle crash injuries: importance of type of crash and occupant role. Accid Anal Prev. 2001;33:167-72.

11. Zhou HH, Liu Q, Yang RT, Li Z, Li ZB. Ocular trauma in patients with maxillofacial fractures. J Craniofac Surg. 2014;25:519-23.

12. Andrews BT, Jackson AS, Nazir N, Hromas A, Sokol JA, Thurston TE. Orbit fractures: identifying patient factors indicating high risk for ocular and periocular injury. Laryngoscope. 2016;126 Suppl 4:S5-11. 